\title{
Development of the Cabernet Autopilot
}

\author{
A. L. Hull, R. E. Strout
}

Prepared by Sandia Laboratories. Albuquerque New Mexica 87115

and Livermore. California 94550 for the United States Energy Research

and Development Administration under Contract AT (29-1) 789

Printed February 1977

When printing a copy of any digitized SAND Report, you are required to update the markings to current standards. 
Issued by Sandia Laboratories, operated for the United States Energy Research and Development Administration by Sandia Corporation.

\section{NOTICE}

This report was prepared as an account of work sponsored by the United States Government. Neither the United States nor the United States Energy Research and Development Administration, nor any of their employees, nor any of their contractors, subcontractors, or their employees, makes any warranty, express or implied, or assumes any legal liability or responsibility for the accuracy, completeness or usefulness of any information, apparatus, product or process disclosed, or represents that its use would not infringe privately owned rights. 


\author{
SAND76-8034 \\ Unlimited Release \\ Printed February 1977
}

\title{
DEVELOPMENT OF THE CABERNET AUTOPILOT
}

\author{
A. L. Hull \\ R. E. Strout \\ Electronics Development Division 8159* \\ Sandia Laboratories, Livermore
}

\begin{abstract}
A unique analog control system was required for the Cabernet vehicle. This system, or autopilot, was designed to control and program the glide attitude of the deployable wing and fin glide bomb. The design was an integrated analog-digital control system. The system was designed with total flexibility so that parameter changes were possible at any time in the Cabernet development program.
\end{abstract}

* This work was done while the authors were members of Advanced Mechanical Division 8158. 
CONTENTS

Page

Introduction

General Concept

11

Autopilot Function Block

Pitch and Yaw Attitude Programs

Roll and Yaw Attitude Control

Pitch Attitude Control

Sideslip Damping Control

Matrix Function

A/D Converters and Look-Up Tables $\quad 22$

Fin Control System $\quad 24$

Telemetry Signal Conditioning, Reference Supplies, and Temperature Monitor

$\begin{array}{ll}\text { FTU-2 System Gain Constants } & 27\end{array}$

$\begin{array}{ll}\text { Autopilot Construction } & 27\end{array}$

$\begin{array}{ll}\text { Testing } & 28\end{array}$

Appendix A--Circuit Schematics 33

$\begin{array}{ll}\text { Appendix B--Drawings } & 37\end{array}$ 


\section{ILLUSTRATIONS}

Figure

Page

1. Cabernet Glide Bomb

2. Application of Mechanical Systems Concept to Electronic Control System in Design of Autopilot

3. Basic Control System for Autopilot

4. Cabernet Attitude Structure

5. Electronic System Design

6. Programmer Design

7. Look-Up Table Design

8. Fin Servo Amplifier Design

9. Completed Autopilot

10. Autopilot Printed Wiring Boards

11. Use of Extender Board During Checkout

12. Interconnection of Wiring Boards in Autopilot Package

13. Fin Angle History for STU-2

14. Roll History for FTU-2 


\title{
DEVELOPMENT OF THE CABERNET AUTOPILOT
}

\author{
Introduction
}

The Cabernet glide bomb program ${ }^{1}$ was initiated to prove the feasibility of deploying an aircraft-launched glide vehicle capable of performing a preprogrammed gliding maneuver. The Cabernet project represented the first step toward the development of a glide bomb which, after being released from a low-flying aircraft, could maneuver itself to a target and allow sufficient escape time for the delivery aircraft. Figure 1 shows the Cabernet vehicle with wings and fins deployed.

The autopilot used in Cabernet is an analog-digital system designed to control the in-flight attitude of an aircraft-released glide bomb having two deployable wings and three deployable fins. To achieve this control, the autopilot commands angular rates and positions to the appropriate fin servo actuator, which changes the vehicle's aerodynamic trim attitude. These trim changes are commanded in a manner to maintain control over the angular velocities and attitude of the vehicle and ultimately the vehicle trajectory.

The Cabernet autopilot must perform a number of functions. It must monitor the vehicle pitch angle, roll angle, yaw angle, pitch rate, roll rate, yaw rate, angle of attack, and sideslip angle. From these parameters it must maintain predetermined roll, pitch, and yaw attitudes by commanding appropriate fin angles. It must provide a steering control system to which the fin angle commands can be channeled. The autopilot must also provide for basic actuation functions, including wing actuate and wing bolt fire signals when it receives verification that stable roll conditions have been achieved. In case of a malfunction or an unstable flight situation resulting in failure of the wings to extend, the autopilot must provide a signal to deploy the recovery parachute. The autopilot also provides a simplified "open loop" guidance system by providing a programmable command history of pitch angle versus time and heading angle versus time. Also included is the capacity to cope with the changing fin effectiveness as a function of angle of attack and sideslip through the use of electronic look-up tables. Finally, it must condition the flight parameters and control data to be telemetered to the ground station for the vehicle posttest performance study.

\footnotetext{
${ }^{1}$ A more detailed description can be found in Cabernet Development Report, M. B. Loll, SAND75-8048, January 1977.
} 


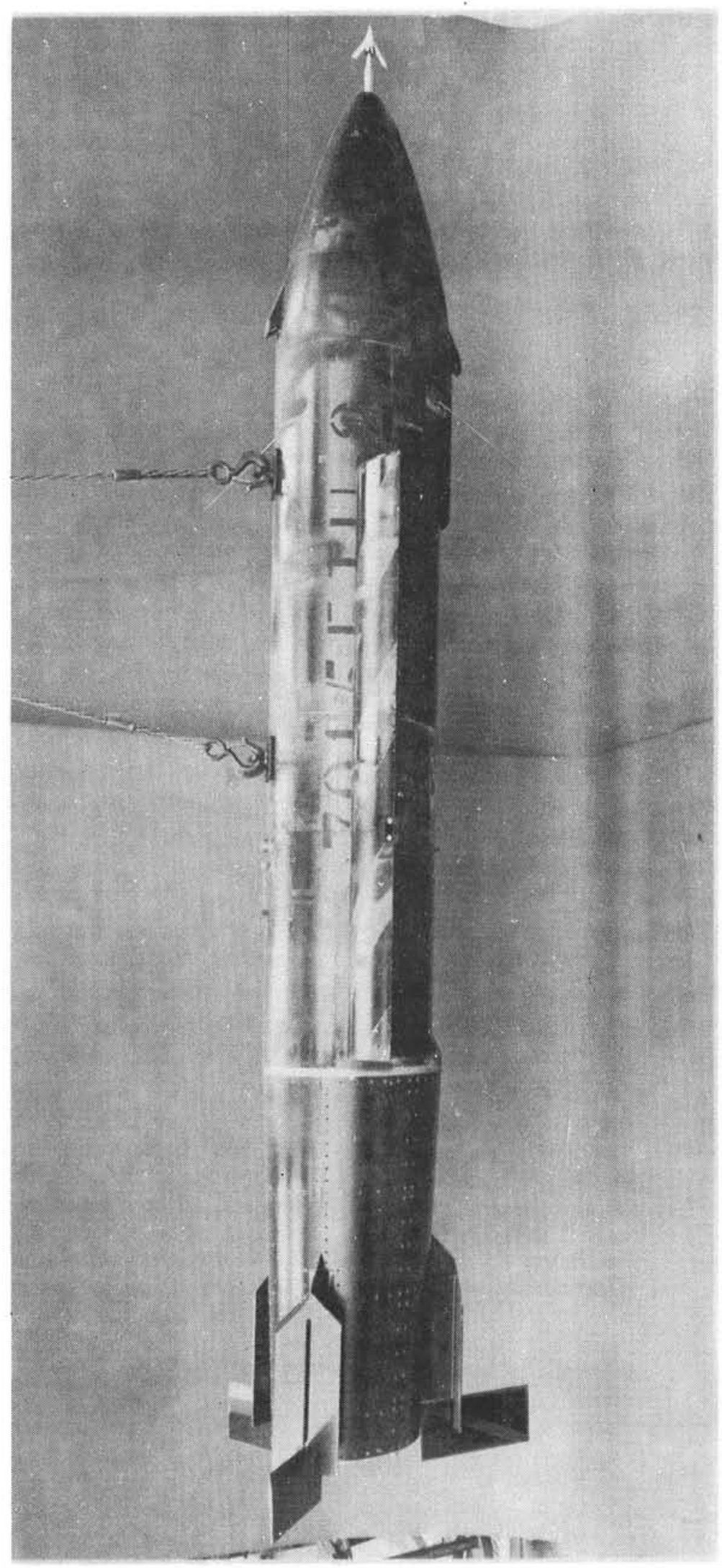

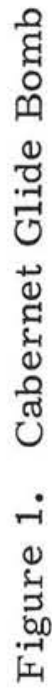


The autopilot must withstand a temperature environment of $-40^{\circ} \mathrm{C}$ to $+55^{\circ} \mathrm{C}$, as well as sled track vibration environments and the less severe aircraft vibration environment. It should require medium or low power to operate.

\section{General Concept}

The design of the Cabernet autopilot involves defining the general controls concept for the glide bomb system in terms of the aerodynamic configuration and the electronic control system. More specifically, in this concept, as shown in Figure 2, the autopilot is fed information from the several flight parameter outputs, the attitude program, and the "pull-out," "fins-up, "and "wings-up" switching functions. From vehicle release time, the autopilot processes this information, actuating the wings or parachute systems according to preset conditions of roll attitude, rate, and time and outputs the appropriate commands to the fin servo control circuits, which in turn position the fins. The fins, which represent the interface between the electronic control system and the aerodynamic forces and moments, modify the vehicle's angular velocity and attitude. To close the control loop, the resultant body attitudes, rates, and trim angles are coupled back to the autopilot through the control attitude reference platform (CARP), the body-fixed triaxial rate gyros, and the angle-of-attack and sideslip nose transducer. In addition, flight and control data are conditioned and channeled to the telemetry system.

The autopilot block of Figure 2 can be viewed in terms of several subsystems, the most important of which is the basic control system illustrated in Figure 3. This basic control system consists of three control loops: roll and yaw control, pitch angle control, and sideslip angle control. Steering is achieved by channeling the error signals from each of these loops through the matrix function block, according to predetermined weighting factors, to the appropriate fin control systems. The control signals from the matrix block to fins one and three are modified at the "look-up table" block to compensate for changes in fin effectiveness at various combinations of angles of attack and sideslip.

The basic Cabernet control system of Figure 3 can be viewed in more detail in terms of the mechanical control system concept, which is presented in the form of the Cabernet attitude structure of Figure 4. The three basic loops can again be seen with the associated program and flight parameter inputs.

The yaw and roll control loop (or heading control) of Figure 4 receives a yaw command $\psi_{0}$ from the heading program and compares this with the actual yaw from the CARP. The error signal is converted to a roll command 


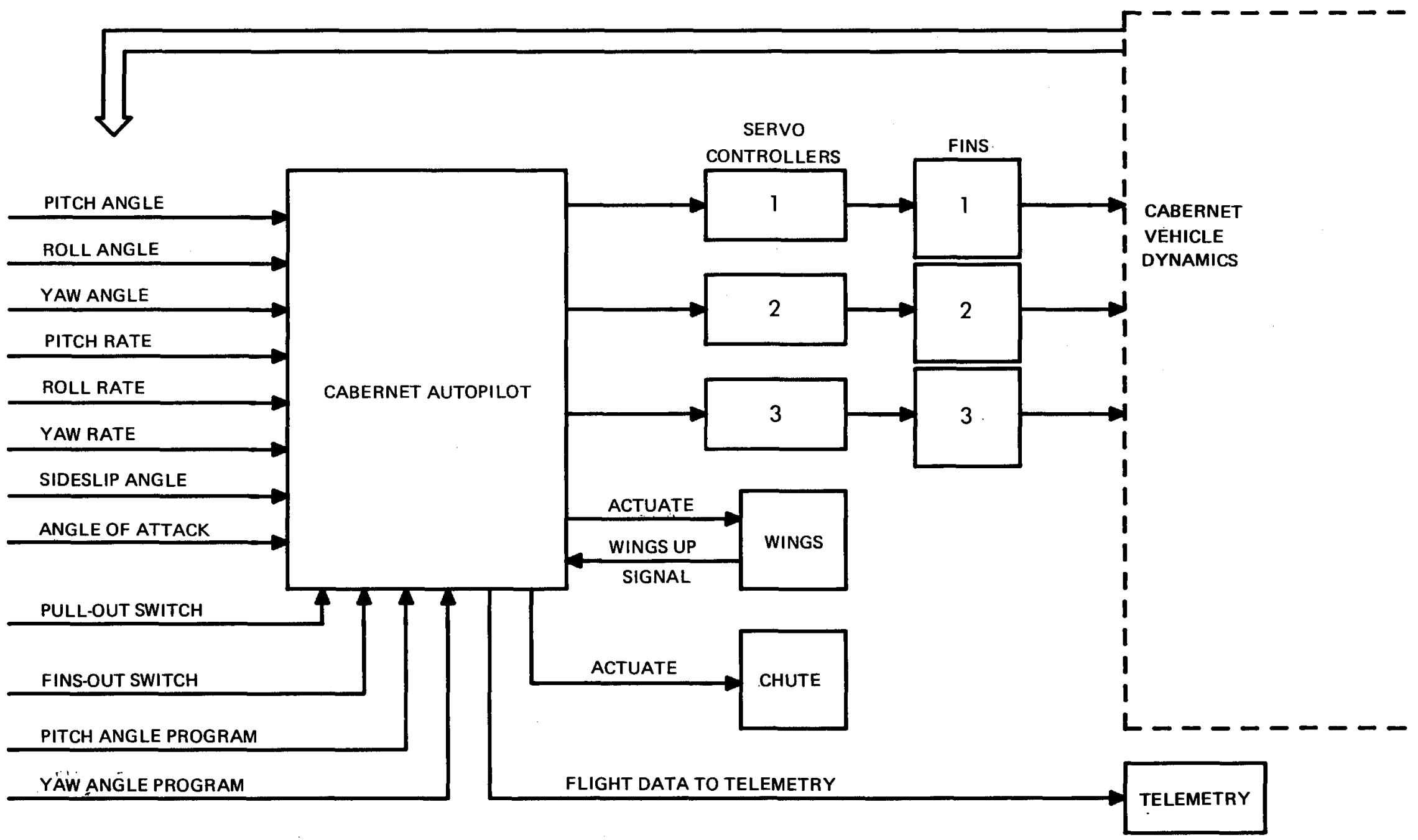

Figure 2. Application of Mechanical Systems Concept to Electronic Control System in Design of Autopilot 


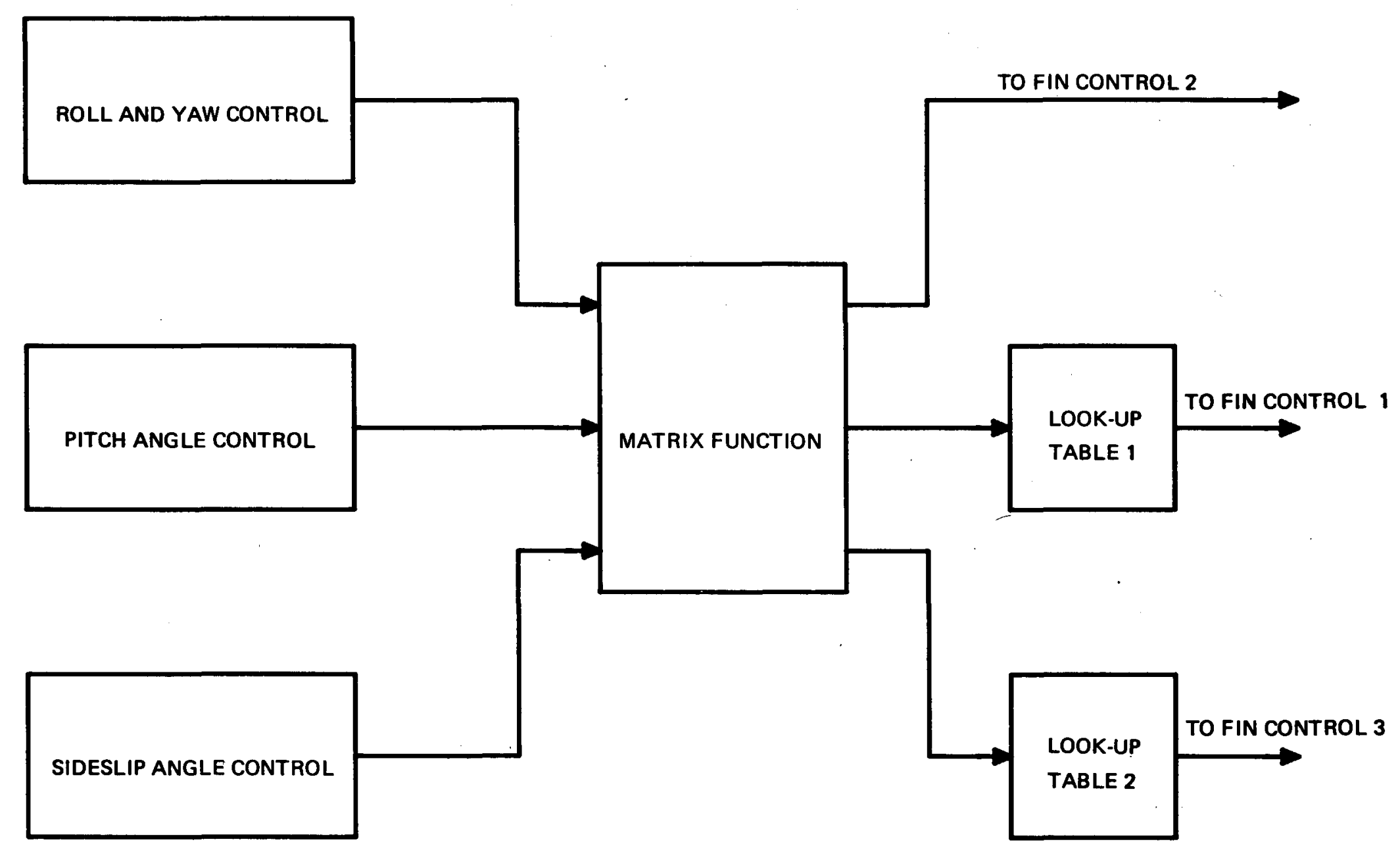

Figure 3. Basic Control System for Autopilot 


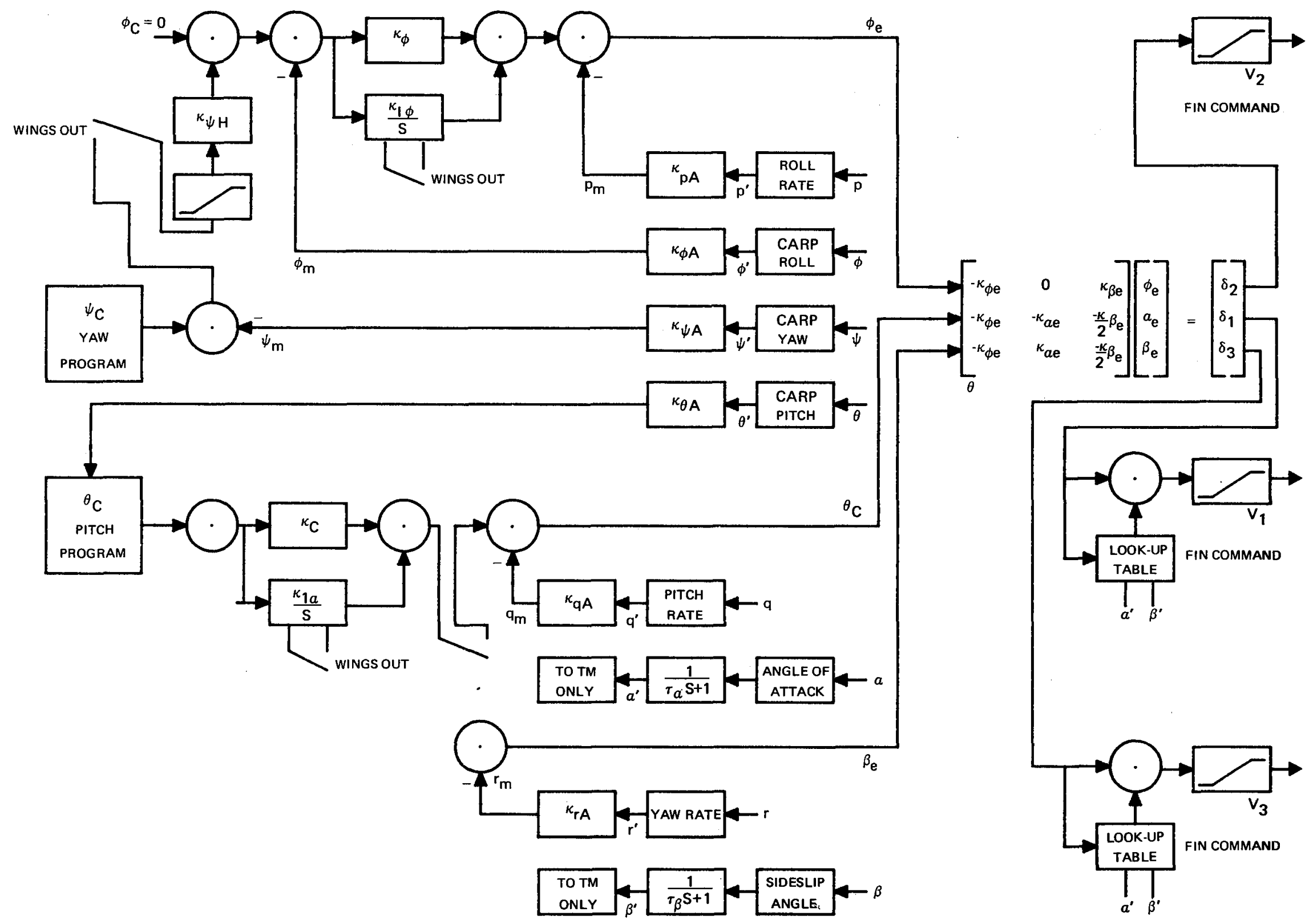

Figure 4. Cabernet Attitude Structure 
which commands the three fins to roll the vehicle. This results in a bankto-turn. When the roll command is achieved and the roll command (yaw error) returns to zero, the vehicle has a net heading change. Roll rate feedback is used in this loop to provide roll damping.

The pitch control loop receives a pitch command from the pitch program which is fed to fins one and three to achieve the desired pitch. At one time this was to be an angle-of-attack control loop, but the pitch angle measurement was felt to be much more reliable than the angle-of-attack transducer, which failed on two vibration tests. The pitch control response is dampened by the pitch rate signal.

Finally, the sideslip angle control was originally designed to provide coordinated turns (bank-to-turn maneuvers), but the previously mentioned failures of this transducer led to the modification of this loop to one of yaw' damping only and depended upon the natural "weather stability" of the vehicle to achieve a coordinated turn. The outputs of the three control loops are summed using various weighting factors. The resultant three-fin command voltages are fed to the fin control systems.

From these basic mechanical concepts, the electronic control system design was formulated. The electronic structure for the entire Cabernet autopilot control system and peripheral systems is shown in Figure 5 . The system was designed with total flexibility, since only a few system gain constants were known at the time of fabrication. Each block of Figure 5 represents an electronic circuit with a specific function as it is organized in the autopilot structure.

Autopilot Function Block

The autopilot function block controls the required timing and switching functions within the autopilot. The sequence of events, some of which are controlled by the autopilot beginning with T-0 release time, is as follows. The autopilot receives the T-0 signal and initiates its timing counter. At the same time, an explosive timer is fired within the Cabernet vehicle to connect the various explosive systems to the firing circuitry. At $T+0.3$ second the telemetry sequencer fires the fin thermal batteries, and at $T+1.0$ second the fins are extended. At $T+1.55 \mathrm{~s}$, the autopilot senses any one of three fin switches at the function block and activates the fin servo control circuits, which begin to return the Cabernet vehicle to zero roll attitude and rate. At $\mathrm{T}+3.0$ seconds the autopilot function block tests for a fins -up signal, $0^{\circ} \pm 10^{\circ}$ roll attitude and $0^{\circ} / \mathrm{s} \pm 30^{\circ} / \mathrm{s}$ roll rate; if all conditions are met, a signal is channeled to the wing capacitor discharge unit (CDU), which in turn fires the wing squib, allowing stored gas pressure to actuate the wings. When the wings have sufficiently extended $\left(120^{\circ}\right)$, the 


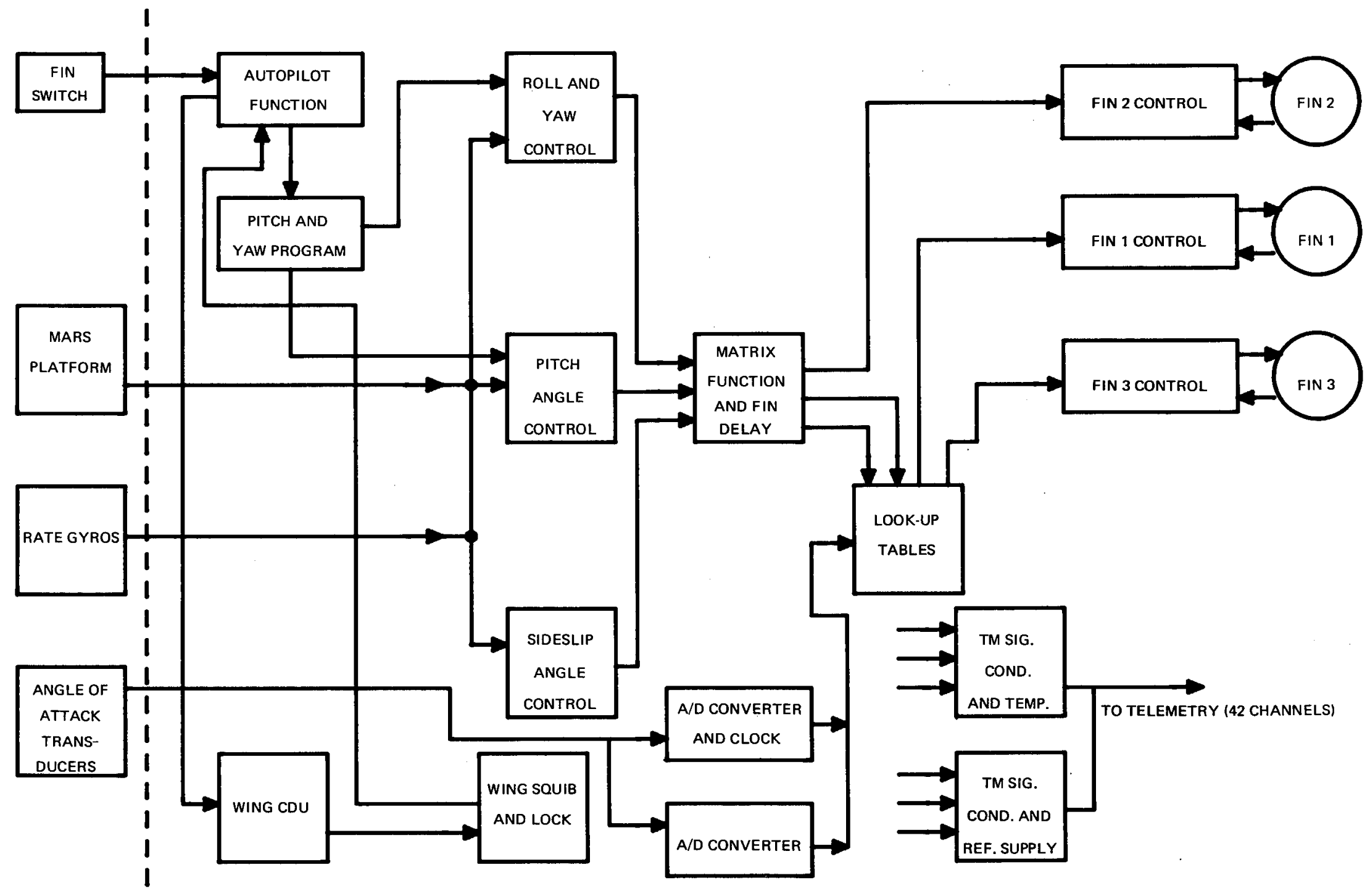

Figure 5. Electronic System Design 
autopilot function block detects the corresponding voltage from a wing dis placement gage. This level causes the various control circuit integrators and signal switches to unlatch. It also initiates the pitch and yaw attitude programs. If at $\mathrm{T}+9.4$ seconds no wing displacement or wing lock signal is sensed by the autopilot function block, the recovery parachute is triggered. Following a successful flight, the program reaches its final attitude step and holds, allowing the telemetry sequencer to fire the recovery parachute to carry the Cabernet vehicle to a safe landing.

The operation of the autopilot function block, shown schematically in Appendix A-3, is as follows:

First, the T-0 signal from the "pull-out" switch enables the autopilot wing timer, A4-U1; the wing timer latch, A5-U3B; the wing fire latch, A5-U3A; the wing fire one-shot, A5-U5; the salvage parachute fire latch, A5-U4A; the program counter, A4-U2; and the clutch enable timer latch, A14-U3. In a normal flight, any one of the three "fin-up" switches will be detected by the "fins-up" gate, A5-U6A, which, along with the clutch enable timer latch, A14-U3, serves to enable the fin servo systems. The fins-up gate, A5-U6A, also serves to set "high" one input of a four-input wing fire "nand" gate, A5-U10A, the other inputs of which are controlled by the "roll angle window" comparator, A5-U1, the "roll rate window" comparator, A5-U2, and the wing timer latch, A5-U3B. Thus, when these four inputs go high, latch A5-U3A is clocked, triggering the monostable A5-U5, which turns on a pair of Darlington transistors, A1 and A2, for one second. Each Darlington fires a wing CDU system to activate a wing squib valve and explosive wing retaining bolt.

As the wings are extended, a wing displacement gage relays the wing position to the autopilot, which detects, through comparator A5-U7, when the wings are opened to 120 degrees. This wings-open comparator initiates the pitch angle and heading program and activates the pitch control loop, yaw control, roll attitude integrator, and pitch attitude integrator. As a backup to the wing displacement transducer, a wing lock switch signal is gated in to perform the same function as the wing displacement comparator. If neither a wing displacement signal nor a wing lock signal is seen within $9.4 \mathrm{~s}$, as determined by counter A4-U1, the salvage parachute latch, A5-U4A, triggers the salvage parachute through transistors A5-Q3 and A6-Q1 and locks out the wing fire signal. 
The pitch and yaw attitude programs determine attitude of the Cabernet vehicle from the time the wings are extended to the time the recovery parachute is deployed. These two programs are initiated by the wings-up signal. Each program has 256 command steps, each step having a duration of $0.1 \mathrm{~s}$. The yaw program provides command levels to the roll and yaw control block, while the pitch program provides command levels to the pitch control block. A block diagram representation of this system is shown in Figure 6.

Each program circuit consists of a 256-by-8-bit programmable readonly memory ( $\mathrm{P} / \mathrm{ROM}$ ) having 256 storage locations, each location addressable through an 8-bit address. The yaw P/ROM, A4-U9 of Appendix A-3, is addressed by a CIMOS ripple counter, A4-U2. The digital data stored at the addressed location in the P/ROMI is fed to a digital-to-analog converter, A4-U11, which is operated in the unipolar mode using an operational amplifier, A4-U6. The analog signal from this amplifier is fed to the yaw command input of the yaw control circuit. The yaw commands can vary from 0 to 30 degrees.

The pitch attitude program is basically the same as the yaw program, except that the pitch $\mathrm{P} / \mathrm{ROM}$ feeds digital data to a digital-to-analog converter, A4-U10, which is connected in a bipolar configuration using two amplifiers, A4-U12 and A4-U13, so that both negative and positive pitch attitudes can be channeled to the pitch command input of the pitch control circuit. The pitch command range is \pm 20 degrees.

\section{Roll and Yaw Attitude Control}

The roll and yaw control block operates in two modes. The first mode, the "fins-up" mode, operates to maintain a zero-degree roll attitude and roll rate. In this mode the roll rate and roll attitude are fed back to the input of the control circuitry in such a way that no error signal results at the output of the system if there are a zero-degree roll attitude and a zero angular velocity.

When the switch is made to the second, or wings-out, mode, two things happen. The roll control loop integrator is activated and the yaw control loop is closed. Thus, a yaw command signal from the yaw program can now be accepted and compared to the actual yaw attitude from the CARP. The resultant error signal is fed into the roll control loop as a roll command, replacing 


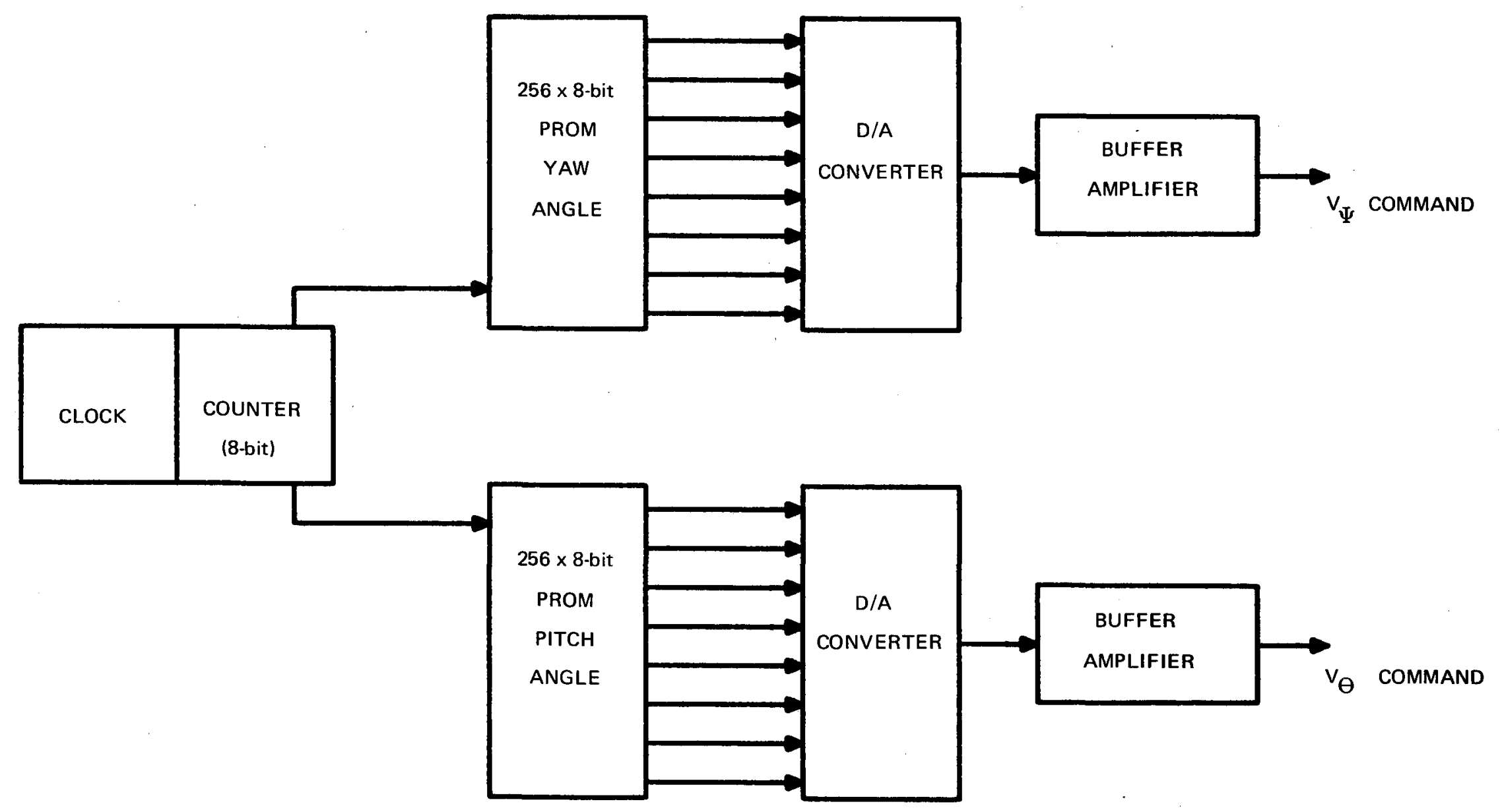

Figure 6. Programmer Design 
the zero degree roll command of mode 1. In effect, a yaw command results in a roll maneuver which, due to the vehicle aerodynamic configuration and the yaw damping control loop, results in a banked turn. This turn is seen as a change in yaw attitude which reduces the magnitude of the resultant yaw command error.

The circuitry used to accomplish this roll and yaw control function can be seen in Appendix A-2. In mode one, the amplifier A8-U6 receives the roll attitude signal from the CARP and filters and buffers the signal to be fed to the telemetry signal conditioning as well as to amplifier A8-U3, which is used in mode 2 as a summing amplifier. The output of A8-U3 is summed into amplifier A8-U4, whose output represents the roll command to be processed to the fin control circuitry.

In mode 2, amplifier A8-U7 receives the yaw attitude signal from the CARP, filters and buffers this signal, and feeds the signal to the telemetry signal conditioning block, as well as to amplifier A8-U8, where it is compared to the yaw attitude command signal from the program block. The resultant yaw attitude error signal from A8-U8 is limited in magnitude to an equivalent $\pm 30^{\circ}$ roll command by back-to-back zeners A8-VR1 and A8-VR2; it is at this point that the mode switching occurs whereby the error signal from A8-U8 is grounded or allowed to pass to the next amplifier stage by the analog switch A6-U1, whose state is controlled by the wingsup gate in the function block. Thus in mode 2 the yaw attitude error signal from A8-U8, with appropriate gain, is compared to the roll attitude signal at amplifier A8-U3. As in mode 1, the output of amplifier A8-U3 is fed to the final summing amplifier A8-U4. In addition, the roll error signal from $\mathrm{A} 8-\mathrm{U} 3$ is fed to the now active integrator A8-U1, whose output is also summed at the input of $\mathrm{A} 8-\mathrm{U} 4$. The function of the integrator is controlled by the analog switch A8-U2, the state of which is determined by the wings-up gate located in the autopilot function block. Thus the integrated roll error is summed with the roll error at amplifier A8-U4. In addition the roll rate signal from the rate gyro is fed to amplifier A8-U5, which serves to filter the signal to an upper frequency of $100 \mathrm{~Hz}$ while removing any dc offsets. This rate signal, with the appropriate gain, is subtracted from the summed signal at the roll command output amplifier A8-U4 to provide sufficient damping of the roll control signal to prevent large overshoot or oscillation.

\section{Pitch Attitude Control}

The pitch attitude control block, like the roll and yaw control, operates in two modes. In the first, or "fins-up, mode, the pitch attitude block 
serves to stabilize the Cabernet vehicle in pitch by providing a pitch control signal which is proportional to the negative of the pitch rate signal. In the second, wings-out, mode, the pitch attitude is compared to the pitch command signal from the program block, and the resulting pitch error signal is summed with the integral of this pitch error signal to provide a new pitch control signal. This new signal is again dampened by the subtraction of a signal proportional to the pitch rate.

The circuit used to control this function is seen in Appendix A-2. In mode 1, amplifier $A 7-\mathrm{U} 6$ receives the pitch rate signal from the pitch rate gyro, filtering this signal and feeding it to the inverting input of the summing amplifier A7-U4. All other circuits on this block are inoperative in mode 1. The output of amplifier A7-U4 represents the pitch control signal.

The pitch control circuit switches to mode 2 when the wings are extended. In this mode, the pitch command signal from the pitch program block is compared to the pitch attitude signal from the CARP, by way of buffer-filter amplifier A15-U5. The resultant error signal is fed through the mode 2 switch A7-U7 to the summing amplifier A7-U4. The error signal is also fed to integrator A7-U3, the function of which is controlled by switch A7-U2. The output of this integrator is also summed at amplifier A7-U4. A pitch control output results which is now proportional to the sum of pitch error and integrated pitch error minus the pitch rate signal, again preventing pitch attitude overshoot.

At one time the system was designed to control angle of attack instead of pitch attitude. Amplifier A7-U5 was designed to filter and buffer the signal from the angle-of-attack transducer. The output of this amplifier is now fed to the telemetry signal conditioning block and the now unused look-up tables.

\section{Sideslip Damping Control}

To prevent the glide bomb from oscillating in the sideslip direction, the body-fixed yaw rate signal is used to generate a damping signal which is fed back to fin control circuitry. Although at one time this system included sideslip angle feedback, transducer reliability considerations have reduced the feedback inputs to body-fixed yaw rate only. The vehicle depends upon natural weather stability to maintain a zero sideslip angle.

The system consists of a filter-buffer stage, A6-U4, which passes the $0.6 \mathrm{~Hz}-\mathrm{to}-15-\mathrm{Hz}$ components of the yaw rate signal to amplifier A6-U3. The output of A6-U3 represents the sideslip damping control signal. Amplifier A6-U5 filters and buffers the sideslip angle transducer signal to be fed to the signal-conditioning block and the look-up tables. 
Matrix Function

The three control signals described in the previous three sections are processed by the matrix function block which steers the vehicle by generating the appropriate fin commands. The roll control signal is directed to each of the three fins equally. The pitch attitude control signal is given equally, but in opposite directions, to fins one and three. Third, the sideslip control signal (yaw rate damping) is fed to fins one and three equally, but to fin two in the opposite direction and with twice the gain.

The circuit for the matrix function board is shown in Appendix A-2. The circuit consists of three summing amplifiers, each of which provides the command voltage for each fin. Amplifier A14-U5 takes the three control signals, applies the appropriate gains, and outputs the fin one command voltage. Fin two commands are generated by amplifier A14-U6, and fin three is commanded by the output of A14-U4.

\section{A/D Converters and Look-Up Tables}

Until late in the Cabernet program, the predicted nonlinearities in the fin aerodynamic responses with various angles of attack $\alpha$ and sideslip angle $\beta$ were of great concern. In particular, the interference by the wings on the airflow pattern at fins one and three was of prime importance. To handle these nonlinearities in fin effectiveness, the analog/digital (A/D) converter and look-up table system was designed.

The system consisted of two 4-bit tracking analog-to-digital converters, as shown in the block diagram of Figure 7. The input of one A/D converter was connected to the angle-of-attack transducer, while the other A/D was connected to the sideslip angle transducer, so that for every combination of angle of attack and sideslip angle there was a unique 8-bit word generated. Each word represented an address on each of two 256-by-8-bit programmable-read-only memories (P/ROMs). The two P/ROMs represented correction data for fins 1 and 3 for various values of $\alpha$ and $\beta$. The data from each of the two P/ROMs were fed into a multiplying digital-to-analog converter which multiplies the fin one or three command voltage from the matrix function block by a weighting factor of from 0 to 1 as determined by the value stored on the P/ROM location, as defined by the particular combination of $\alpha$ and $\beta$. This product is then added to the original command voltage times a gain factor. Thus this new value of fin command voltage is fed to the fin one or three control system; the fin two command is left unchanged as it comes from the matrix function block. 


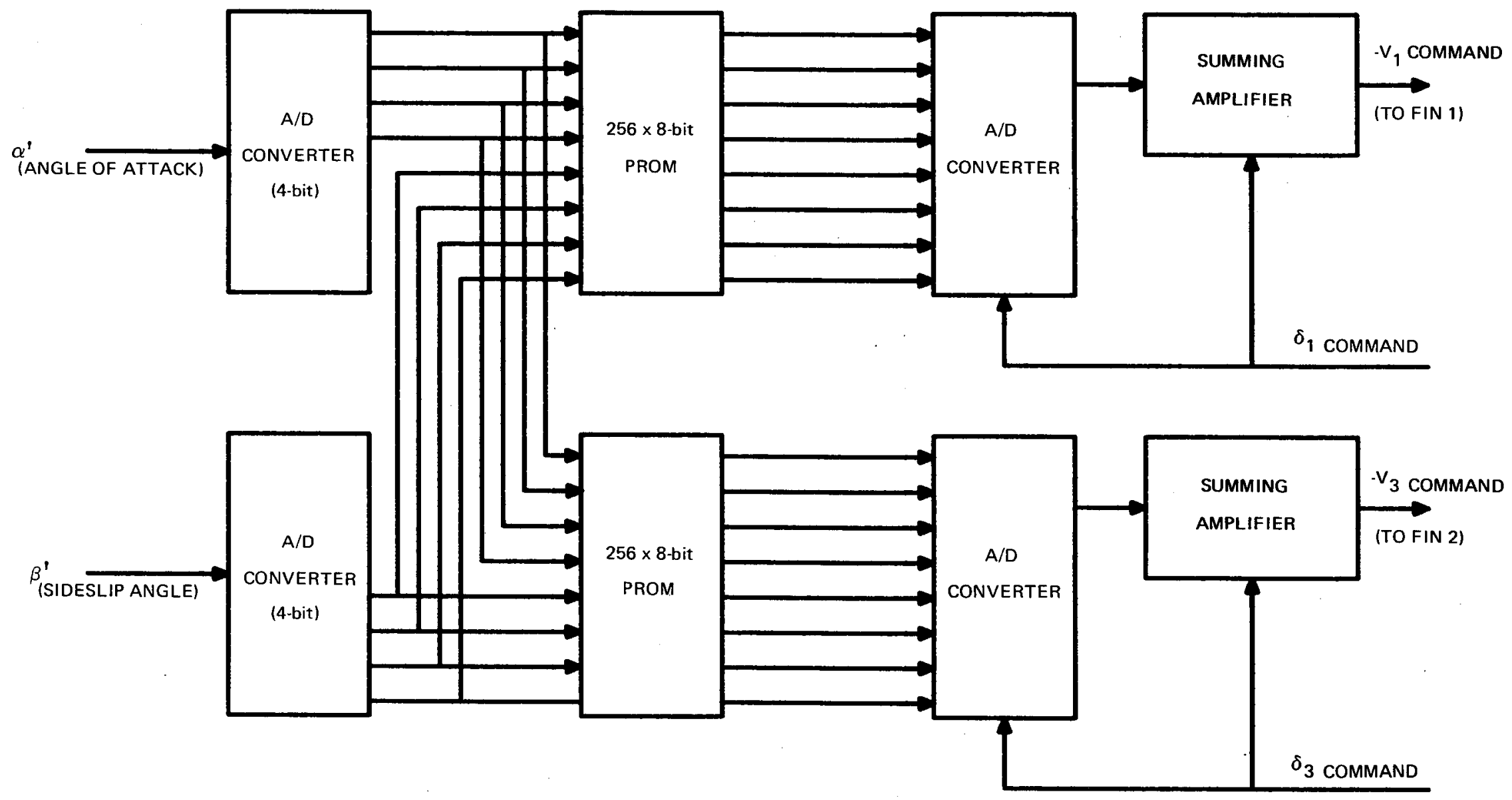

Figure 7. Look-Up Table Design 
The circuit for the two A/D converters and the look-up tables is shown in Appendix A-3. The signal from the angle-of-attack transducer buffer amplifier, described earlier, enters the A/D converter $A 1$ at the input of amplifier A1-U6. At this amplifier the signal from a digital-to-analog (D/A) converter, A1-U4 driven by an up/down counter, A1-U3, is compared to the angle-of-attack input signal. The difference signal is fed to comparators A1-U1, which determine which direction the up/down counter should count in order to match the two signals; oscillator A1-U8 clocks the counter. When the two signals are equal, gates $\mathrm{A} 1-\mathrm{U} 2$, in conjunction with the flipflops A1-U7, stop the counter. Thus at this point the digital count from the four-bit counter represents the digital equivalent of the analog input signal from the angle-of-attack transducer. A similar four-bit digital output is obtained from the sideslip angle A/D converter circuit A2.

The two four-bit words form an eight-bit address which is fed to each of two 256-by-8-bit P/ROM look-up tables A3-U1 and A3-U2. The 8-bit digital output of each $\mathrm{P} / \mathrm{ROM}$ is fed to a digital-to-analog (D/A) converter, A2-U3 and A2-U4, respectively. Each D/A converter takes the product of the analog equivalent of the 8-bit digital input and the fin command signal from the matrix function block. This product is summed with a signal proportional to the fin command signal at amplifier A2-U6 and A2-U7. This sum results in a new fin command voltage, corrected according to variations in $\alpha$ and $\beta$.

In the final autopilot configuration, the look-up table function was not used because of lack of confidence in the nose transducer used to obtain values of $\alpha$ and $\beta$. It was felt that adequate vehicle response could be obtained without the advantage of look -up table compensation.

Fin Control System

Each fin command voltage must now be converted to an actual fin deflection. This occurs in the fin control system. In this system the fin command voltage representing a particular desired fin angle is compared to a voltage representing the actual fin angle, as shown in Figure 8 . The difference in these signals, the error voltage, drives two parallel amplifier stages, one stage for positive fin angle error and the other stage for negative fin angle error. One amplifier stage drives the counterclockwise magnetic clutch and the other amplifier drives the clockwise magnetic clutch, thus allowing for control in both directions with a unidirectional, continuously operating motor. 


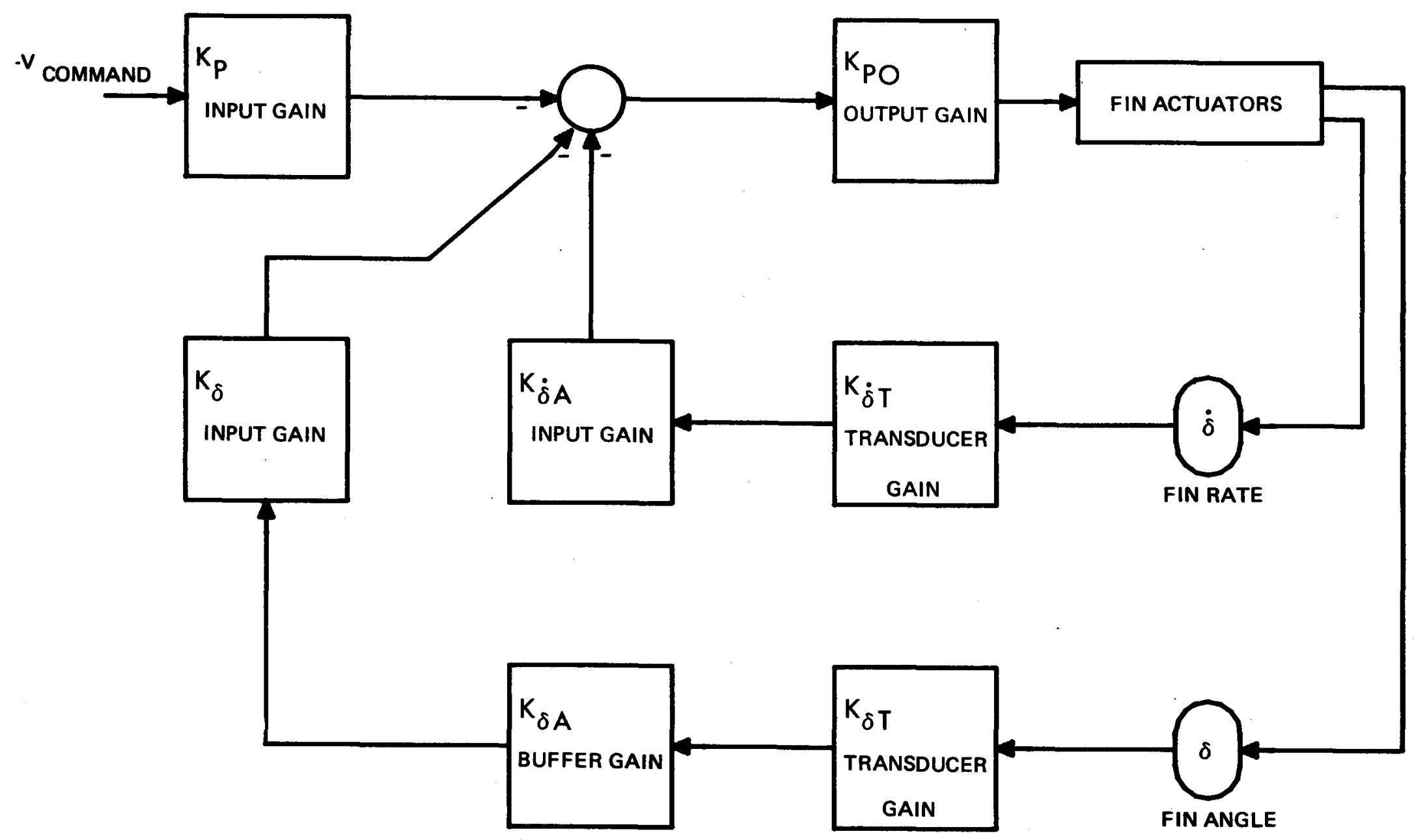

Figure 8. Fin Servo Amplifier Design 
The fin control circuit can be seen in Appendix A-2, block A9. The fin command voltage is fed to the input of summing amplifier A9-U5, through resistors $\mathrm{R} 19$ and $\mathrm{R} 20$. At the junction of the resistors, the command voltage is electronically limited to $\pm 9 \mathrm{~V}$ by zener diodes VR3 and VR4. This corresponds to a command limit of \pm 20.25 degrees. At the summing am plifier A9-U5, the command voltage is compared to a voltage proportional to the sum of the fin acceleration and position. The fin position is obtained from the voltage follower A9-U6, the input of which ties to the center tap of the fin position potentiometer. The command error signal obtained from the output of A9-U5 is split off to separate amplifier stages A9-U7 and A9-U4. Amplifier A9-U7 is the driving amplifier for the positive direction fin deflection, and amplifier A9-U4 is the driving amplifier for the negative direction fin deflection. Each amplifier drives a high-power Darlington transistor, A9-Q1 or A9-Q2, which activates the appropriate magnetic clutch for fin deflection in the desired direction. Analog switches A9-U2 and U3 provide for deactivation of the fin control system until full fin deployment. The fin deflection clutches are two magnetic particle clutches located in the fin servo actuator assembly, mounted in the aft section near the fins.

Basically, the fin servo actuator assembly consists of a high-speed DC motor which engages a ball screw type of linear actuator by one of the two clutches described above. The linear motion of the actuator is converted to angular motion of the fin through a bell crank assembly.

\section{Telemetry Signal Conditioning, Reference Supplies,} and Temperature Monitor

The signal-conditioning block conditions the several signals representing flight parameters and autopilot diagnostics, making them compatible with the inputs of the telemetry system. In addition, a temperature monitor within the autopilot is situated in this block. The three reference supplies, +10 volts, -10 volts, and -9 volts are also located in this block.

The circuits for this block are shown in Appendix A-1. Regulators A16-U1, A16-U2, and A16-U3 are the three reference sources. The several resistors $A 16-R 18$ through $R 61$ represent the dividers used to convert the \pm 10 -volt signals from the autopilot outputs to 0 - to 5 -volt signal levels required by the telemetry inputs. The temperature monitor is represented by A15-U6 and its associated components. A15-U4 is the scaling amplifier for the high gain roll channel. Amplifiers A15-U1, U2, and U3 are the three differential amplifiers which sense the voltages from the servomotor current viewing resistors (CVRs) and condition them accordingly to be fed to the telemetry inputs. The various voltage monitors are fed to the telemetry through A15-R35, R36, and R37. 


\section{FTU-2 System Gain Constants}

The electronic control system was adjusted to provide the required overall gain constants as determined by an analog computer simulation of the GLADD system. The gain constants and definitions are shown below:

$$
\begin{aligned}
\mathrm{K}_{\mathrm{qa}} & =\frac{\text { Fin Deflection }}{\text { Pitch Rate }}=\frac{0.58 \text { degree }}{\text { degrees/second }} \\
\mathrm{K}_{\theta} & =\frac{\text { Fin Deflection }}{\text { Pitch Angle Error }}=\frac{0.77 \text { degree }}{\text { degree }} \\
\mathrm{K}_{\mathrm{I} \theta} & =\frac{\text { Fin Deflection }}{\text { Integrated Pitch Error }}=\frac{0.20 \text { degree }}{\text { degrees } / \text { second }} \\
\mathrm{K}_{\mathrm{pa}} & =\frac{\text { Fin Deflection }}{\text { Roll Rate }}=\frac{0.15 \text { degree }}{\text { degrees } / \text { second }} \\
\mathrm{K}_{\phi} & =\frac{\text { Fin Deflection }}{\text { Roll Angle Error }}=\frac{0.2142 \text { degree }}{\text { degree }} \\
\mathrm{K} & =\frac{\text { Roll Command }}{\text { Yaw Angle Error }}=\frac{8.0 \text { degrees }}{\text { degrees }} \\
\mathrm{K}_{\mathrm{I}} & =\frac{\text { Fin Deflection }}{\text { Integrated Roll Error }}=\frac{0.033 \text { degree }}{\text { degrees } / \text { second }} \\
\mathrm{K}_{\mathrm{ra}} & =\frac{\text { Fin Deflection }}{\text { Yaw Rate }}=\frac{3.0 \text { degrees }}{\text { degrees } / \text { second }} \\
\mathrm{K}_{\text {Fin }} & =\frac{\text { Fin Deflection }}{\text { Fin Command Voltage }}=\frac{22.5 \text { degrees }}{10 \text { volts }}
\end{aligned}
$$

Autopilot Construction

There were several constraints on the construction of the autopilot. The sled and aircraft environments to which the autopilot would be subjected necessitated a sturdy package and special connectors to withstand severe vibration. The nature of the design required flexibility for making lastminute parameter changes, so plug-in type printed wiring boards were used. 
Because of the short development time, standard "off-the-shelf" components were required. In addition, the circuit boards were to be packaged on a "space available" basis, even though the circuits and system requirements had not been completely defined at the time at which space was allotted.

Standard printed wiring board techniques were used in designing the autopilot, shown in completed form in Figure 9. The case design allowed the use of up to 16 printed wiring plug-in type cards, as shown in Figure 10. The connectors used on the cards were the same type as those used in the proven design of a PCM at SLA; environmental testing had shown the connectors to have very stable contacts during vibration and shock environments. The plug-in printed wiring card was chosen to allow access to cards during setup and checkout of the autopilot through the use of an extender-board, as shown in Figure 11.

After all circuit boards had been set up and checked out, a conformal coat of Silgard 184 was applied to the front and back of each board to protect the components during vibration environments. The Silgard 184 was also applied to the base of the printed wiring board mating connectors, coating the solder cups to prevent any small metallic particle from creating a short between any of the connector pins. The back of the connector can be seen in Figure 12. All components had to withstand a wide temperature range, i. e., $-40^{\circ} \mathrm{C}$ to $55^{\circ} \mathrm{C}$, such as might be seen during flight and ground tests.

Testing

The purpose of the Cabernet testing was to prove the feasibility of controlling the glide trajectory of a deployable wing and fin vehicle. No attempt was made to provide a complete guidance and control system.

Two tests of the Cabernet vehicle incorporated the autopilot. The first test, STU-2, was run primarily to prove the adequacy of the wing and fin systems under the anticipated aerodynamic loads. This test consisted of the Cabernet vehicle, mounted upside down, with the nose tilted slightly to the ground (to simulate aerodynamic loading), on a rocket-propelled sled attached to rails. During the test the wings and fins were actuated and a fin wiggle program was initiated. The autopilot commanded the fins through various angles, thus testing fin response under the actual aeroloads which might be experienced in flight. All electronic systems performed adequately on this test, as shown in the plot of fin angle versus time (Figure 13). As can be seen in this figure, the larger angle deflections were more difficult to achieve. 


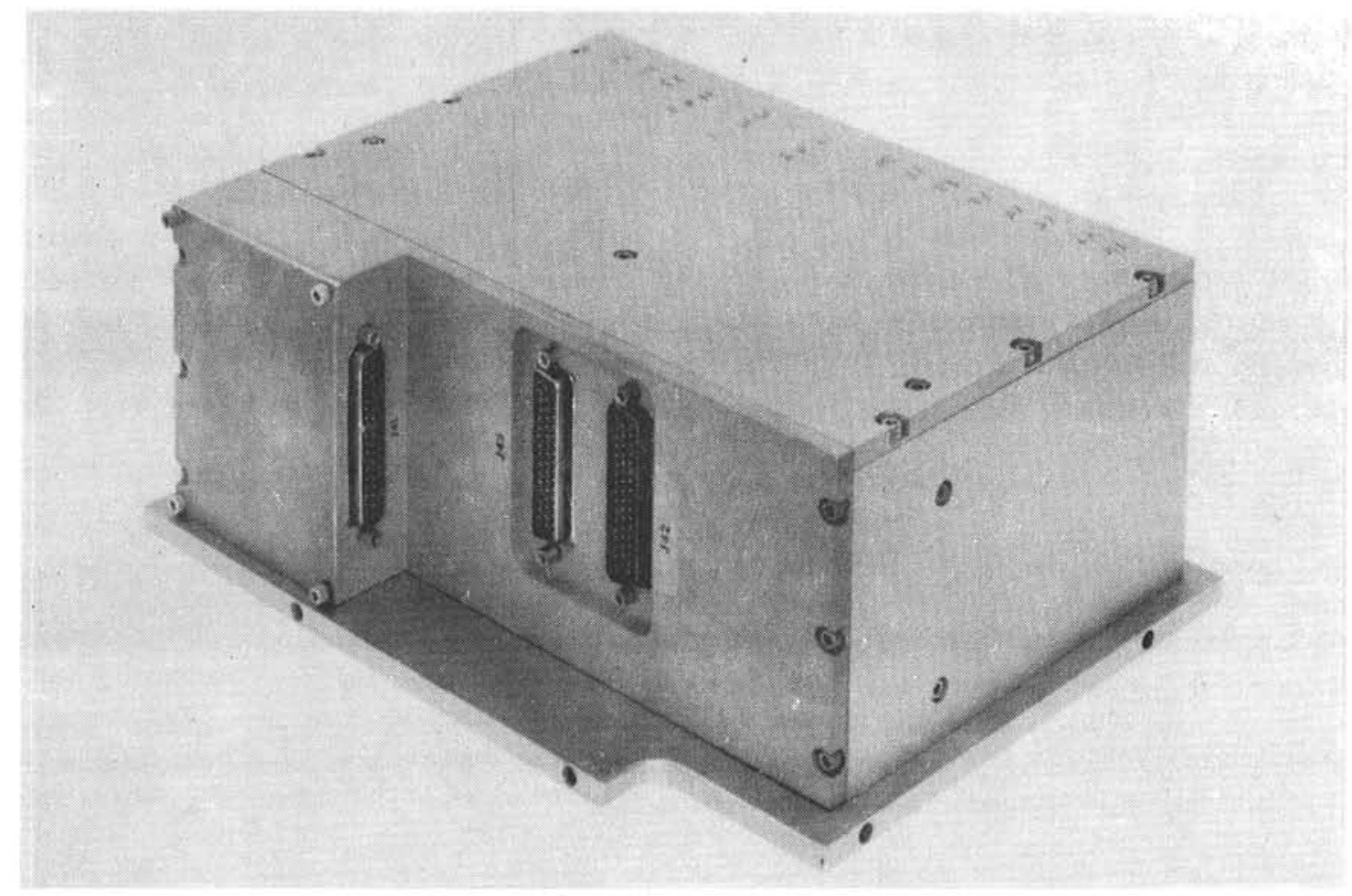

Figure 9. Completed Autopilot

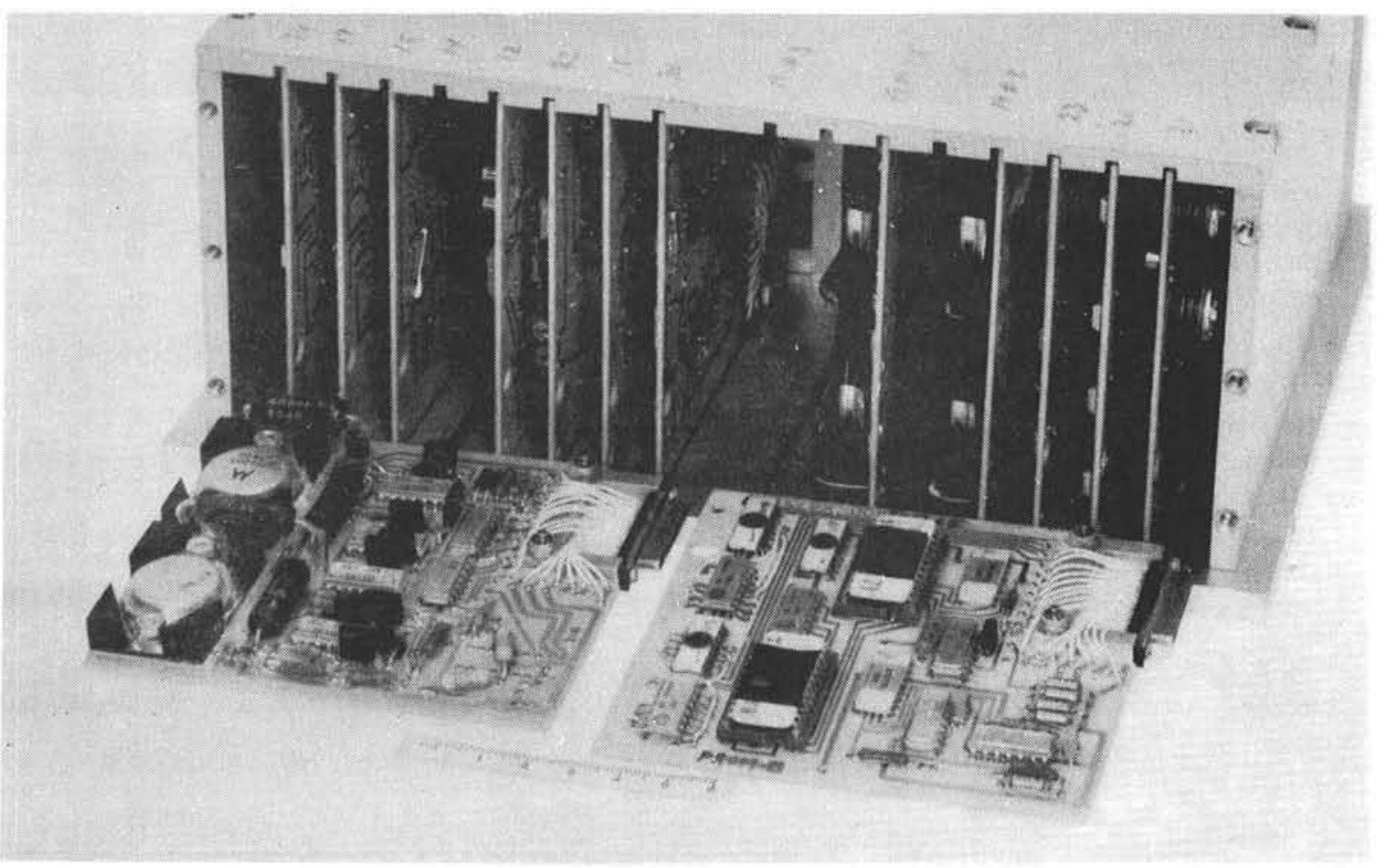

Figure 10. Autopilot Printed Wiring Boards 


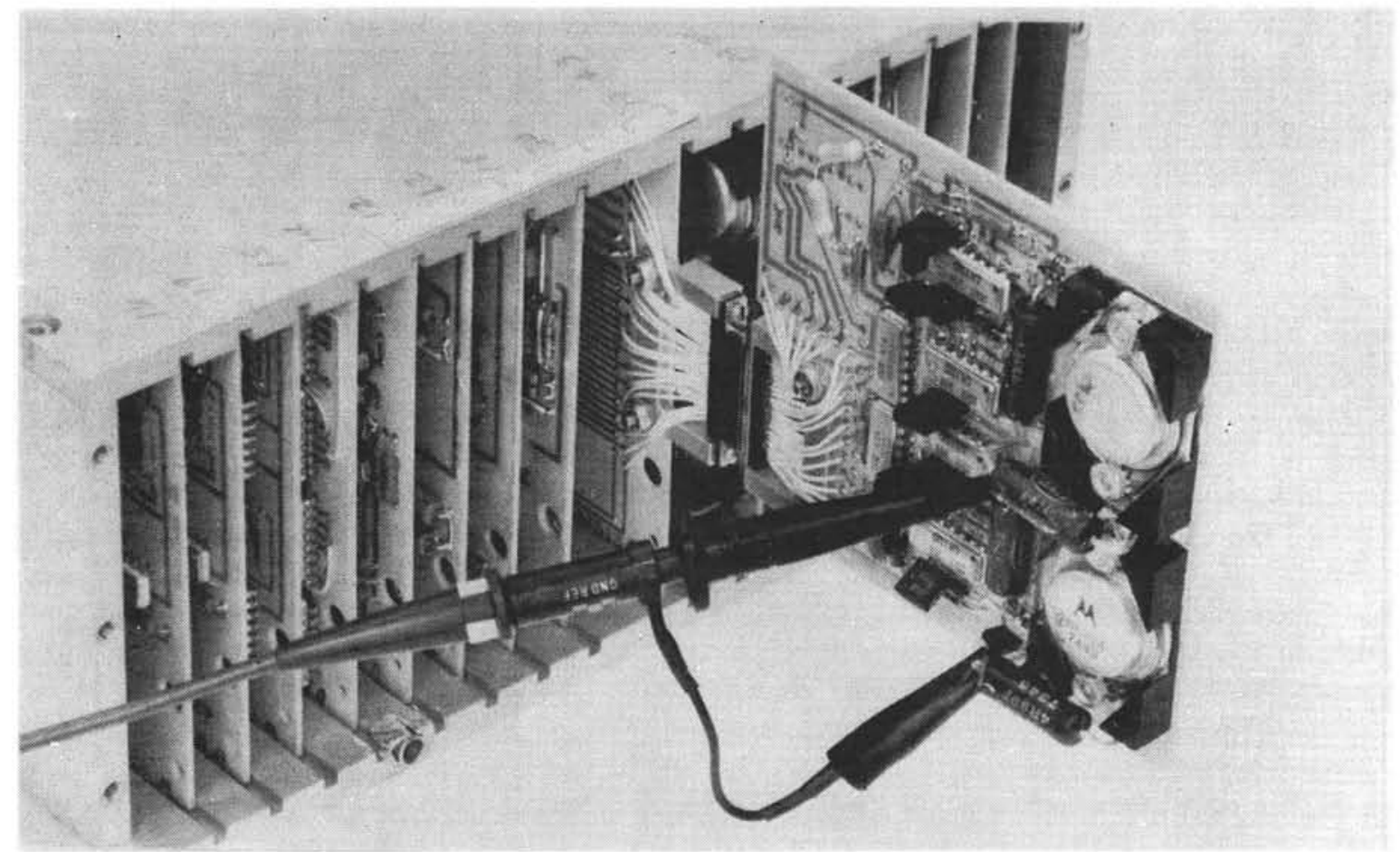

Figure 11. Use of Extender Board During Checkout

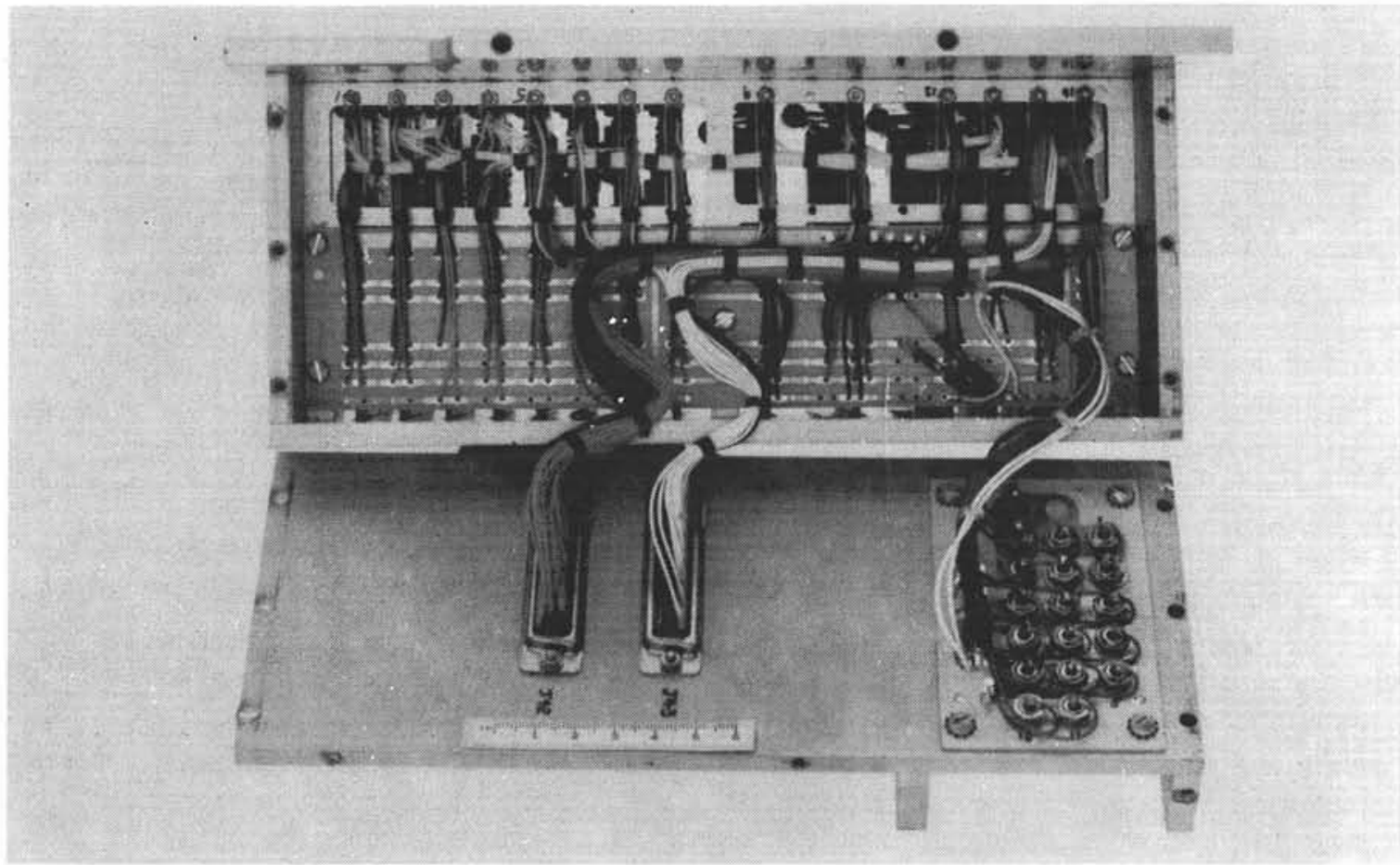

Figure 12. Interconnection of Wiring Boards in Autopilot Package 


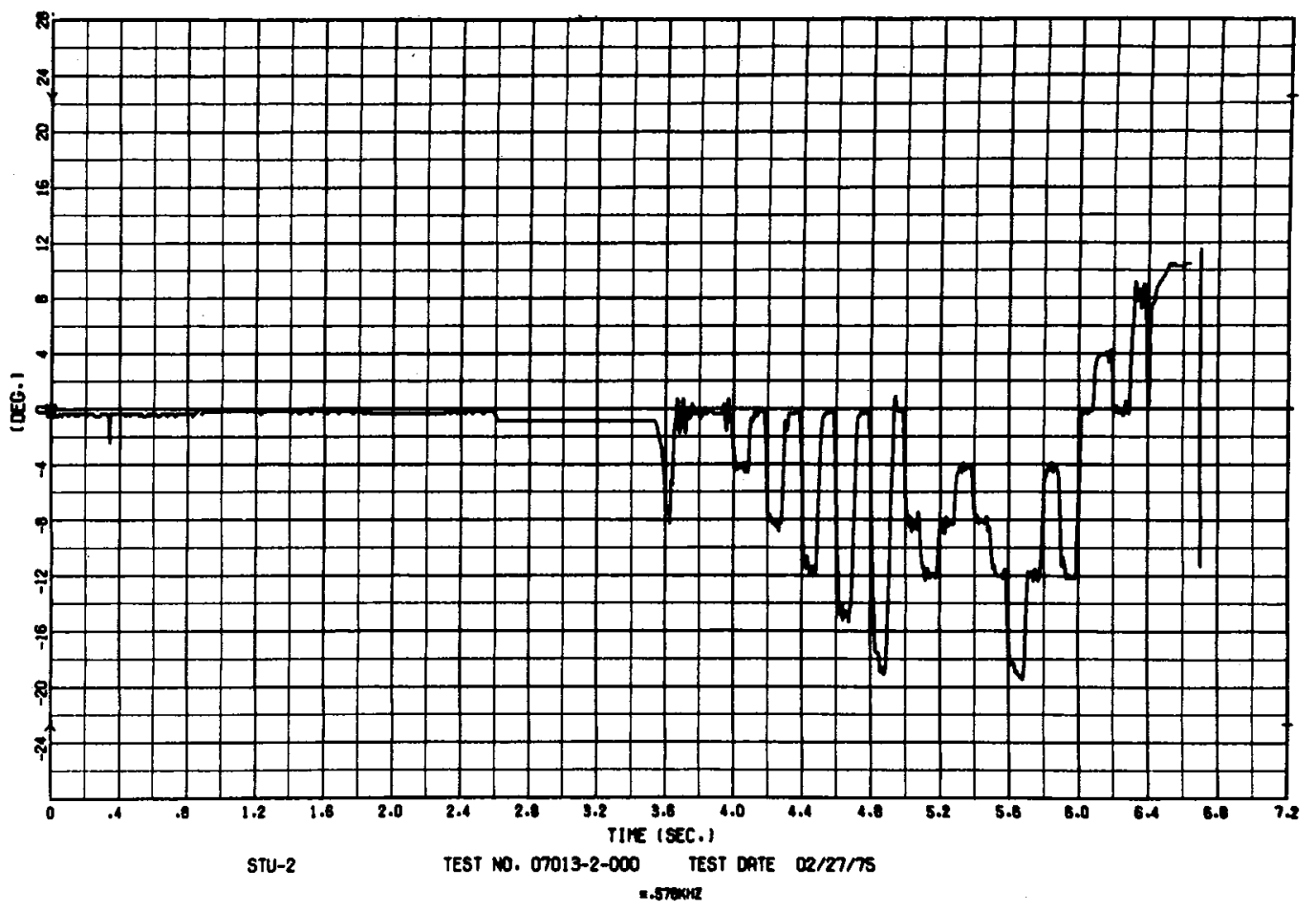

Figure 13. Fin Angle History for STU-2

Following this test some preliminary Flight Dynamics Laboratory (FDL) simulations were conducted to gain confidence in the adequacy of the autopilot design to maintain control of the Cabernet vehicle. The tests consisted of mounting the autopilot and the associated control attitude reference platform on the FDL three-axis rate platform. Nearby were placed three fin servos which were torsion bar-loaded to simulate aerodynamic loading of the fin. The actual fin angles were fed to an analog computer in which was programmed the aerodynamic model of the Cabernet vehicle. The analog computer, in turn, fed commands to the three-axis rate table based on its calculations of how the vehicle would respond to the given fin deflections. This simulation proved very useful, as many control parameters were more readily defined. In essence, the full flight test could be simulated at the FDL with actual hardware without the risks or expense of an actual flight test.

The final test was a full system test, FTU-2, which involved releasing the Cabernet vehicle and allowing it to perform a preprogrammed maneuver identical to that simulated in the FDL. Upon release of the vehicle from the aircraft, thermal batteries were initiated which energized the fin servo motors. Unfortunately, the magnetic particle clutches in one of the servo actuators had become jammed because of compaction of the clutch particles, leaving one fin inoperable. This problem resulted in very minimal control of the vehicle, which consequently impacted the ground before the parachute was deployed. The roll versus time plot is shown in Figure 14 for this test. 


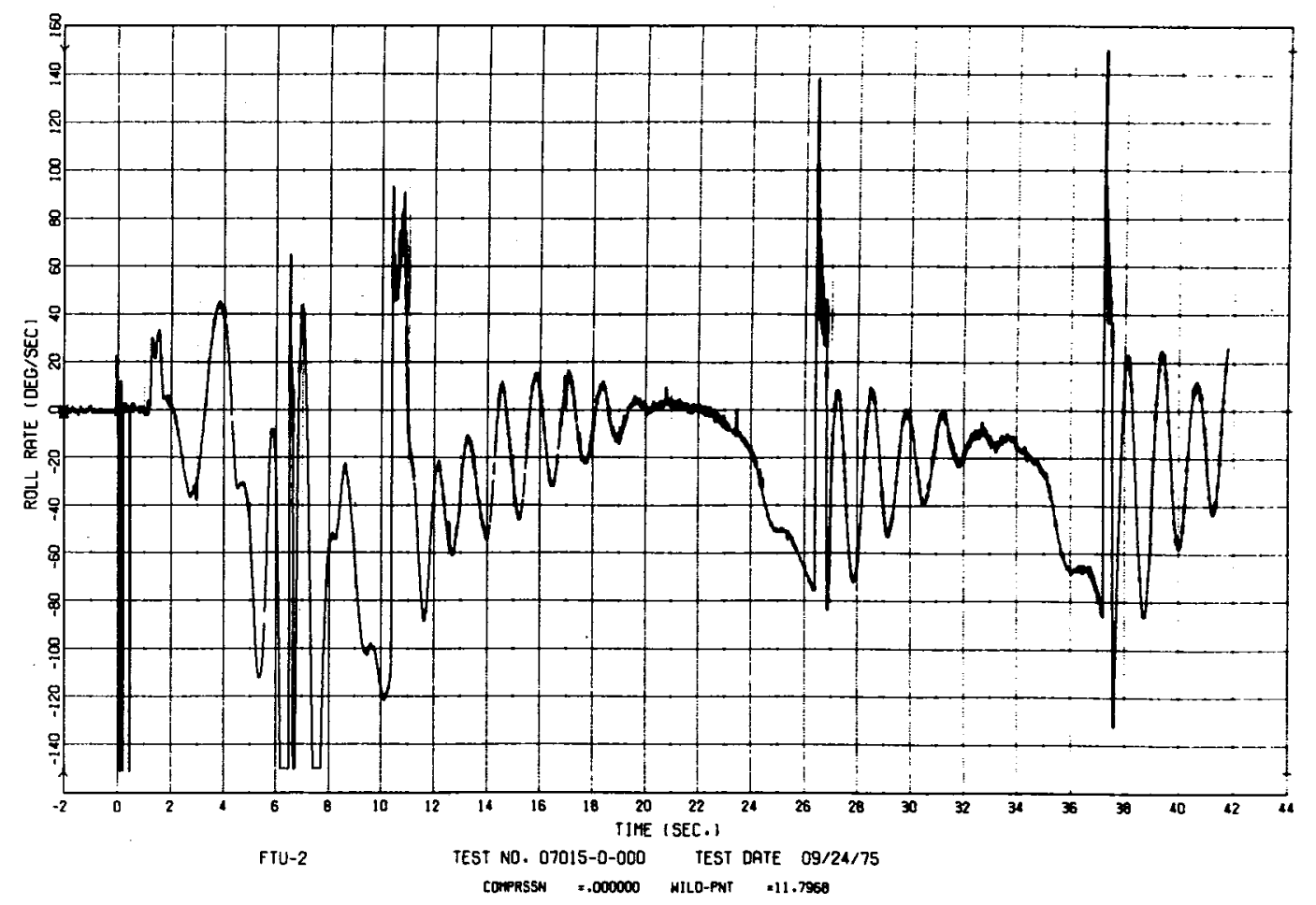

Figure 14. Roll History for FTU-2

In this and earlier tests the autopilot performed accurately in achieving the proper control functions of the Cabernet vehicle. Although program changes made FTU-2 the last Cabernet test, sufficient data were obtained to allow the conclusion that the testing techniques were valid and that such a controlled glide bomb is feasible. 
APPENDIX A--CIRCUIT SCHEMATICS 


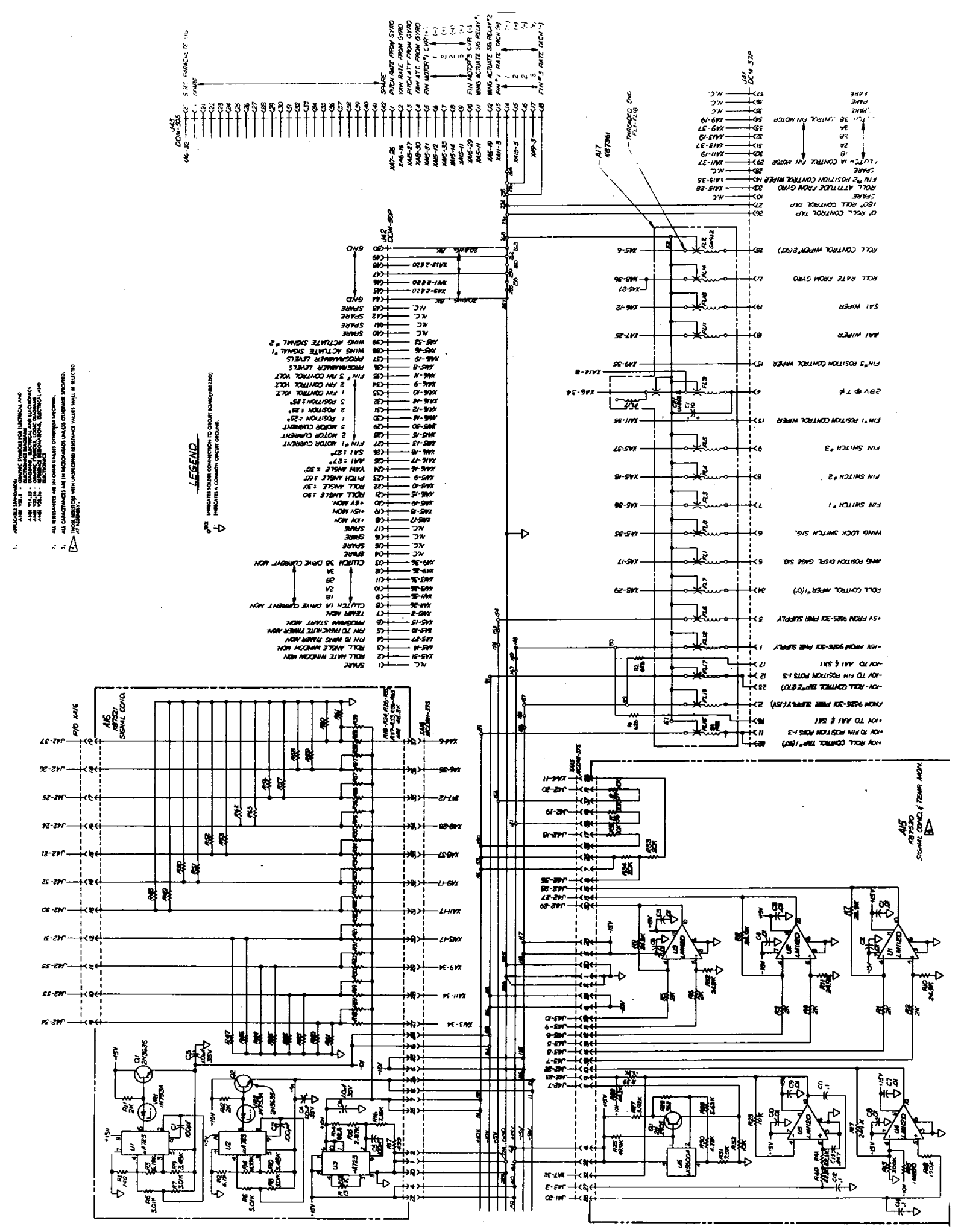




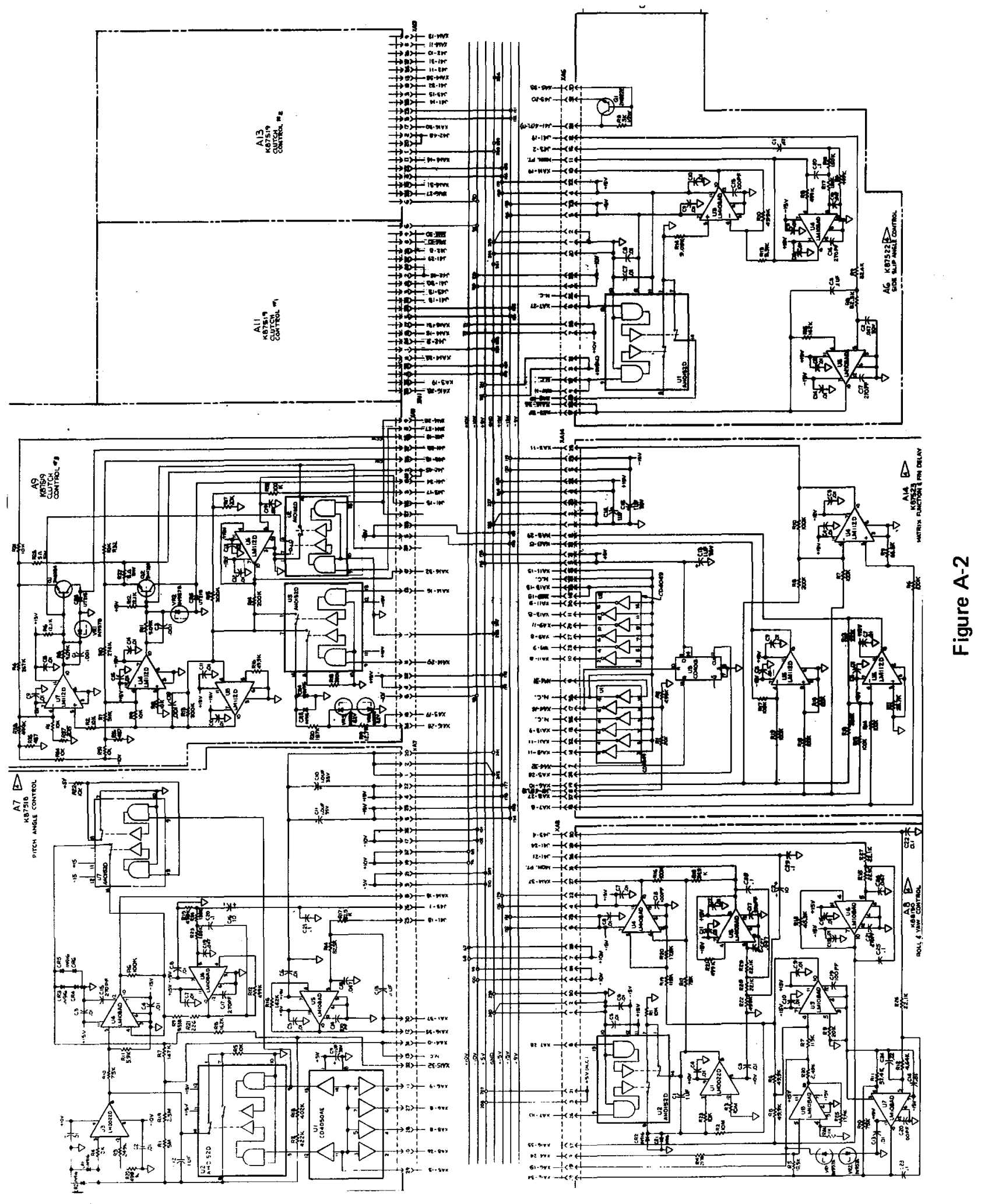




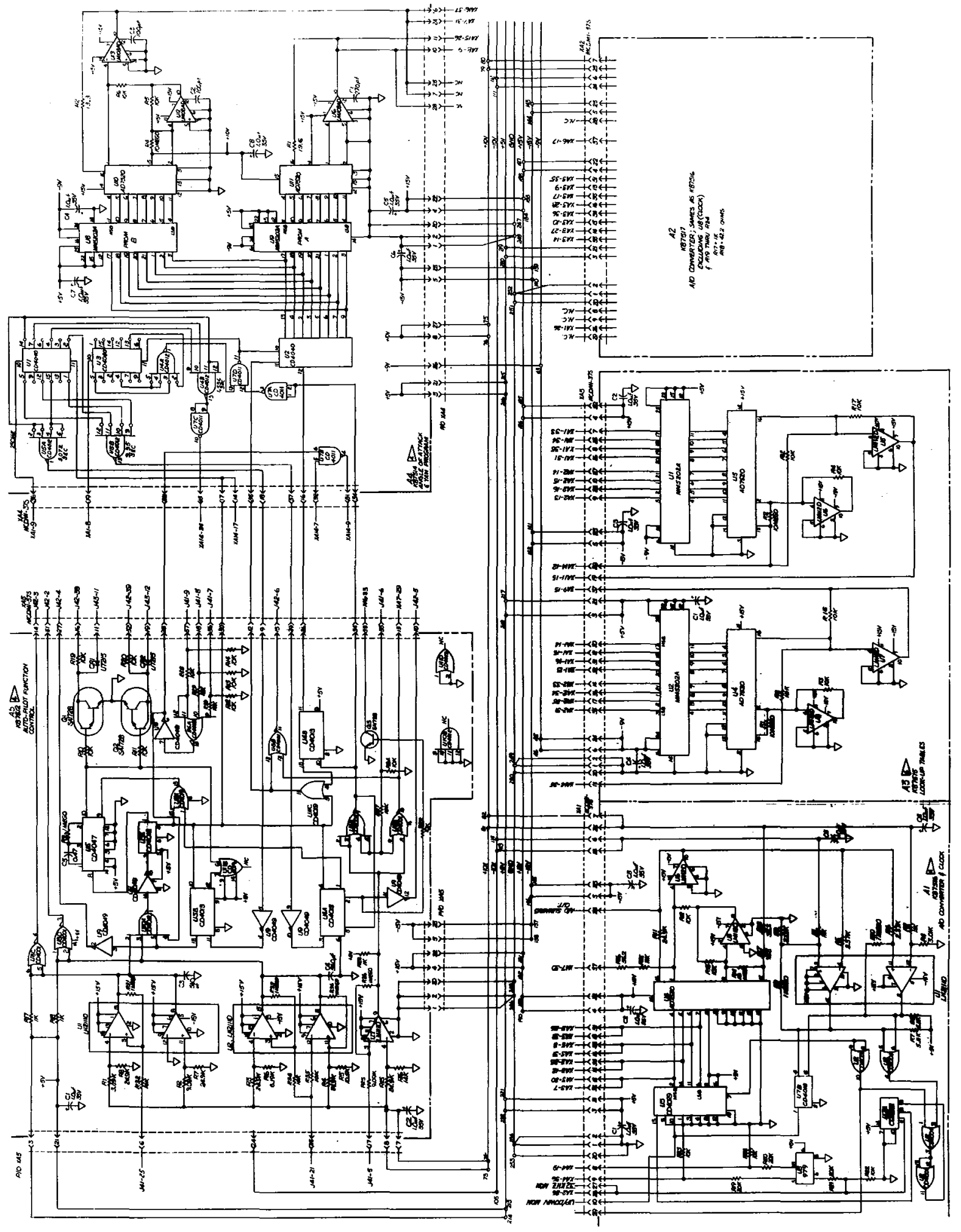

管 
APPENDIX B--DRAWINGS 
1. K87305

2. K87306

3. K87307

4. K87308

5. K87309

6. K87310

7. K87311

8. K87312

9. K87313

1.

2. CMK87512

3. NCK87512

4.

5. CMK87513

6. NCK87513

7. K87514

8. CMK87514

9. NCK87514

10.

K87515

11. CMK87515

12. NCK87515

13. K87516

14. CMK87516

15. NCK87516

16.

17. CMK87517
Bracket, connector

Cover

Plate, filter

Plate, side

Plate, right side

Plate, rear

Plate, top

Plate, support

Plate, connector

Autopilot Electrical

Printed wiring assembly

Control master

Numeric control

Printed wiring assembly

Control master

Numeric control

Printed wiring assembly

Control master

Numeric control

Printed wiring assembly

Control master

Numeric control

Printed wiring assembly

Control master

Numeric control

Printed wiring assembly

Control master 
18. NCK87517

19. K87518

20. CMK87518

21. NCK87518

22. K87519

23. CMK87519

24. NCK87519

25. K87520

26. CMK87520

27. NCK87520

28. K87521

29. CMK87521

30. NCK87521

31. K87522

32. CMK87522

33. NCK87522

34. K87523

35. CMK87523

36. NCK87523

37. K88130

38. CMK88130

39. NCK 88130

40. K88230

41. CMK88230

42. NCK 88230

43. K88107

44. CK84757
Numeric control

Printed wiring assembly

Control master

Numeric control

Printed wiring assembly

Control master

Numeric control

Printed wiring assembly

Control master

Numeric control

Printed wiring assembly

Control master

Numeric control

Printed wiring assembly

Control master

Numeric control

Printed wiring assembly

Control master

Numeric control

Printed wiring assembly

Control master

Numeric control

Printed wiring assembly

Control master

Numeric control

Heatsink, transistor

Schematic (FTU-2 only)

NOTE: Drawings 1-42 are for STU-2 printed wiring boards only. 
Wing CDU System

$\begin{array}{rrl}\text { 1. } & \text { K87356 } & \text { Wing actuator, CDU } \\ \text { 2. } & \text { CK87356 } & \text { Schematic, CDU } \\ \text { 3. } & \text { K87357 } & \text { Case } \\ \text { 4. } & \text { K87358 } & \text { Plate, connector } \\ \text { 5. } & \text { K87359 } & \text { Plate, end } \\ \text { 6. } & \text { K87360 } & \text { Printed wiring assembly } \\ \text { 7. } & \text { CMK87360 } & \text { Control master } \\ \text { 8. } & \text { NCK87360 } & \text { Numeric description } \\ \text { 9. } & \text { K87361 } & \text { Printed wiring assembly } \\ \text { 10. } & \text { CMK87361 } & \text { Control master } \\ \text { 11. } & \text { NCK87361 } & \text { Numeric description }\end{array}$




\section{UNLIMITED RELEASE}

\section{INITIAL DISTRIBUTION}

M. L. Kramm, 1320

C. E. Dalton, 1321

R. C. Maydew, 1330

H. R. Vaughn, 1331; Attn: H. R. Spahr

R. G. Clem, 1350; Attn: G. W. Stone, 1351

G. M. Heck, 2326

T. B. Cook, Jr., 8000; Attn: C. H. DeSelm, 8200

B. F. Murphey, 8300

L. Gutierrez, 8100

W. C. Scrivner, 8400

A. N. Blackwell, 8110

W. E. Alzheimer, 8120; Attn: C. S. Hoyle, 8122

W. D. Zinke, 8123

D. E. Gregson, 8150; Attn: J. B. Wright, 8156

G. N. Beeler, 8157; Attn: D. A. Clarin

A. S. Rivenes, 8158

W. R. Bolton, $8158 /$ R. E. Humphrey, 8158

L. A. Hiles, $8158 / \mathrm{M}$. H. Rogers, 8158

D. N. Tanner, 8159

A. L. Hull, 8159

R. E. Strout, 8159

R. D. Cozine, 8160

C. S. Selvage, 8180

R. I. Peterson, 8183; Attn: H. D. Sorenson

D. M. Schuster, 8310

T. S. Gold, 8320; Attn: G. S. Brown, 8324

G. W. Anderson, 8330

J. L. Wirth, 8340

J. F. Barham, 8360

J. D. Gilson, 8366

R. A. Baroody, 8410

S. G. Cain, 8412

R. M. Hargreaves, 8413

A. J. Clark, 9330; Attn: D. C. Bickel, 9335

Technical Publications and Art Division, 8265, for TIC (2)

F. J. Cupps, 8265/Technical Library Processes Division, 3141

Technical Library Processes Division, 3141 (2)

Library and Security Classification Division, 8266-2 